\title{
Medical Reconciliation as a Patient Safety Strategy
}

\author{
Shinu Kuriakose* \\ Associate Professor School of Health Professions New York Institute of Technology \\ *Corresponding author: Kuriakose S, Associate Professor School of Health Professions New York Institute of Technology. \\ To Cite This Article: Shinu Kuriakose. Medical Reconciliation as a Patient Safety Strategy. Am J Biomed Sci \& Res. 2021 - 14(3). AJBSR. \\ MS.ID.001993. DOI: 10.34297/AJBSR.2021.14.001993.
}

Received: 㭗September 21, 2021; Published: 阱 October 05, 2021

\begin{abstract}
Patient Safety has increasingly been receiving attention over the last couple of decades due to a combination of legal suits against hospitals due to negligent care, which could have been avoided, and an increased awareness in the medical community that steps can be taken to mitigate issues which could compromise a patient's health. The Joint Commission on Accreditation of Healthcare Organizations (JCAHO), an accrediting non-governmental body that accredits the vast majority of hospitals and other medical facilities in the United States on the quality of the medical standards, has listed comprehensive medication reconciliation as one of its 2015 National Patient Safety Goals [2]. JCAHO further states, "There is evidence that medication discrepancies can affect patient outcomes."
\end{abstract}

Keywords: Patient safety, Medication reconciliation, Discharge planning, Medication errors, and disability

\section{Introduction}

Medication reconciliation is intended to identify and resolve discrepancies it is a process of comparing the medications a patient is taking (and should be taking) with newly ordered medications. The comparison addresses duplications, omissions, and interactions, and the need to continue current medications. The types of information that clinicians use to reconcile medications include (among others) medication name, dose, frequency, route, and purpose [1]. Organizations should identify the information that needs to be collected to reconcile current and newly ordered medications and to safely prescribe medications in the future." [2]. The article this author chose was published in 2013 in the Annals of Internal Medicine, Janice Kwan and his colleagues estimated that $67 \%$ of patients admitted to hospitals have unintended discrepancies in the medications started in the inpatient units [3]. These patients often were on a different medication regimen before their hospital admission and notwithstanding the medications started for specific hospitalized indications; these patients were also discharged on the wrong medication protocol.

\section{Discussion}

The recommendation, which Kwan described as being the most successful in reducing the percentage of unintended medical errors, was called best possible medication history (BPMH). This process involved a clinician conducting a structured interview with a competent patient and eliciting their medication used (both prescribed and over the counter including herbal supplements, vitamins, and any home remedies) while also confirming this information from a reliable source such as a pharmacy and/or the patient's outpatient primary care physician [3]. The completion of this process involved a second phase which was called the best possible medication discharge plan (BPMDP); a process involving a clinician examining the medicines given to the patient in the hospital and transferring these medications (if indicated by the inpatient medical team) to the patient's pharmacy and relaying this information to the patient's outpatient primary provider while also sitting with the patient and going over all these medications to ensure that the patient understood them [3].

As a clinically practicing physician assistant, this author has often noted that time is the limiting factor, which leads clinicians to cut corners, and not fully, follow through with the medication reconciliation process as documented in most hospital policy manuals. Additionally, patients are poor historians during the initial day of their hospitalizations, as they are in a weakened state, both mentally and physically, due to their illness. Furthermore, 
there are patients with only limited resources, who are often lacking a primary care provider and/or do not fill prescriptions at a regular pharmacy and thus are not able to take benefits of a robust medication reconciliation process. Finally, the increased use of electronic medical record systems have ironically led to less patient face time as often the medications are electronically sent to a pharmacy and physician on record in the system but which the patient probably does not use anymore, thus depriving the advantages of comprehensive medication reconciliation [4].

\section{Conclusion and Recommendations}

While medication reconciliation is mandatory in the United States for accreditation purposes, Kwan and his colleagues discovered that the process by which it was done was not standard and this led to main inadvertent discrepancies. Their study showed that the best results, which led to the lowest errors, were conducted by pharmacists and were the most cost-effective at $\$ 3200$ per 1000 prescription orders [3]. An interesting finding of the study was that although there were such high percentages of medical reconciliation errors, the clinical significance in causing medical harm to patients was quite limited. The authors did state that further studies would be needed to conduct to fully understand this aspect of care.

Kwan and his colleagues recommended that patient safety protocols must encompass initiatives like medication reconciliation during both admission and discharge of patients from hospitals and other medical institutions and there would be value in standardizing this process. Innovative approaches like standardized checklists, holding hospitals responsible for errors in medication reconciliation system failures to include financial penalties might make this process an integral part of the complete patient care experience.

\section{References}

1. Becerra A (2019) Impact of Medication Reconciliation Programs on Reducing Readmission Rate and Enhancing Medication Safety During Transitions of Care. The Permanente Journal.

2. 4- Joint Commission (2018, January). 2019 National Patient Safety Goals.

3. Kwan JL, Lo L, Sampson M, Shojania KG (2015) Medication Reconciliation During Transitions of Care as a Patient Safety Strategy. Annals of Internal Medicine 158(5): 397.

4. Martin CM (2018) Avoiding Errors During Transitions of Care: Medication Reconciliation. The Consultant Pharmacist 27(11): 764-769. 